\title{
LEVANTAMENTO DAS PLANTAS MEDICINAIS UTILIZADAS EM BAIRROS DE SANTA MARIA - RS
}

Nádia Somavilla

Aluna - Especialização em Biologia - Centro de Ciências Naturais e Exatas

UFSM - Santa Maria, RS

Thais Scotti do Canto-Dorow

Departamento de Biologia - Centro de Ciências Naturais e Exatas

UFSM - Santa Maria, RS

\section{RESUMO}

Um levantamento realizado nos bairros de Santa Maria, RS, no ano de 1994, revelou que a população faz uso contínuo de plantas medicinais. Este trabalho foi baseado em entrevistas feitas junto aos moradores de sete bairros da cidade: Nossa Senhora de Lourdes, Medianeira, Nossa Senhora das Dores, Centro, Nossa Senhora do Rosário, Itararé e Patronato. Foram registradas 148 espécies, pertencentes a 134 gêneros, distribuídas em 64 famílias. Como resultado, são apresentadas listas contendo as espécies citadas nos questionários, acompanhadas de nome vulgar, família, indicação terapêutica e farmacógeno.

\section{ABSTRACT}

A survey done in the districts of Santa Maria, RS, revealed that people make continuous use of medicinal plants. This study was based on interviews made with the residents of seven districts of the city: Nossa Senhora de Lourdes, 
Medianeira, Nossa Senhora das Dores, Centro, Nossa Senhora do Rosário, Itararé and Patronato. A total of 148 species were registered, belonging to 134 genus and arranged in 64 families. Lists are shown containing the species mentioned in the questionaries, along with the popular name, family, therapeutic indication and pharmacogeno.

\section{INTRODUÇÃO}

As plantas vêm sendo utilizadas como auxiliares no tratamento de doenças há milhares de anos, por praticamente todos os grupos étnicos. Porém, nas últimas décadas, este uso aumentou consideravelmente, devido a muitas causas e apresentando como consequência, muitos problemas. Como causas principais podem ser citadas: o baixo poder aquisitivo da população; o difícil acesso à assistência médica e farmacêutica; os altos custos dos produtos industrializados; a crença de que os produtos naturais não apresentam efeitos colaterais em relação aos produtos sintéticos; a tendência das pessoas procurarem uma vida mais saudável (SIMŐES et alii, 1989). Desta procura pela cura com base nos produtos de origem natural, resultou a utilização indiscriminada de muitas plantas, principalmente pelo fato de que plantas diferentes podem apresentar o mesmo nome vulgar, sendo tratadas igualmente pela população, proporcionando o manuseio errado destas plantas com relação ao preparo e administração das mesmas.

Devido aos fatores citados acima e outros, é de fundamental importância o estudo etnobotânico e etnofarmacológico, com a finalidade de buscar informações e experiências na população, porém, deve-se aproveitar a oportunidade para esclarecer os equívocos existentes sobre o uso ou manejo das plantas medicinais. O objetivo principal deste trabalho é realizar um levantamento das plantas utilizadas por uma parcela da população santamariense, verificando a indicação terapêutica, a procedência das plantas e as partes da planta que são usadas no preparo da medicação. 


\section{MATERIAL E MÉTODOS}

Para a realização deste trabalho utilizou-se o seguinte procedimento: elaboração do questionário, escolha da amostra e entrevistas.

\section{Elaboração de questionário.}

Através de um levantamento bibliográfico, foram selecionados alguns trabalhos relacionados com a área de etnobotânica. Baseando-se nestes, elaborou-se 0 instrumento da pesquisa, ou seja, uma ficha (FIGURA 1) com questões relativas ao conhecimento de plantas medicinais: nome popular, farmacógeno (parte da planta utilizada) e a forma de utilização. A procedência ou local de obtenção das plantas usadas e os casos de intoxicação com as mesmas, também foram identificados.

\section{QUESTIONÁRIO}

LOCAL DE COLETA (bairro, vila):

U.F.: RS CIDADE: Santa Maria

ENDEREÇO:

1- Costuma usar plantas no tratamento de doenças?
$(\mathrm{)}$ às vezes
( ) sempre
( ) nunca

2- Onde são obtidas as plantas medicinais que utilizas?
( ) em casa
( ) farmácia
( ) amigos
( ) ervateiros

3- Já teve casos de intoxicação (envenenamento) por plantas na família?
( ) $\operatorname{sim}$
( ) não

4- Cite algumas plantas que costuma usar.

a- Nome(s) da(s) planta(s):

b- Para o quê usas?

c- Parte(s) utilizada(s) (farmacógeno):

d- Forma(s) de utilização: 


\section{Amostragem:}

Tomou-se como população alvo, os bairros próximos ao centro da cidade de Santa Maria. O número de bairros foi obtido pela amostragem polar, isto é, foi determinado um ponto central no mapa da cidade, no caso, o Calçadão, localizado à rua Dr. Bozano, e à partir deste, foi traçado um círculo como referência para a retirada dos bairros que serviriam de amostra, totalizando sete (7) bairros: $N^{a} S^{a}$ de Lourdes, Medianeira, $N^{a} S^{a}$ das Dores, Centro, $N^{a} S^{a}$ do Rosário, Itararé e Patronato.

Foram aplicados quatrocentos e quatro (404) questionários. Este número foi determinado utilizando-se um intervalo de confiança de $95 \%$ e um erro de amostragem de $5 \%$. Na escolha do número de ruas por bairro foi utilizada a amostragem aleatória simples.

Quanto ao número de residências, utilizou-se a amostragem sistemática, sendo selecionadas de acordo com o tamanho do bairro. O critério utilizado para considerar bairro grande e pequeno, foi o número total de ruas em cada bairro. Desta maneira, foram aplicados quarenta (40) questionários em cada um dos três (3) bairros considerados pequenos como: Bairros Nossa Senhora de Lourdes, Medianeira e Nossa Senhora das Dores, e setenta (70), em cada um dos quatro (4) bairros considerados grandes: Bairros Patronato, Centro, Itararé e Nossa Senhora do Rosário.

\section{Entrevistas.}

Os questionários foram aplicados à população diretamente pelo pesquisador. Nos locais onde a planta não foi identificada, esta foi coletada e levada ao laboratório da UFSM para identificação. As plantas que não puderam ser identificadas, foram excluídas do presente trabalho.

4. Elaboração dos resultados das entrevistas.

Os resultados obtidos dos 404 questionários aplicados, foram distribuídos em três gráficos (Figuras 2, 3 e 4) e em duas tabelas, contendo as 
seguintes informações: nome popular, nome científico e família das plantas medicinais (Tabela 1); indicação terapêutica e farmacógeno das plantas medicinais em estudo (Tabela 2).

Os nomes populares foram mencionados com base em nomes utilizados pela população, durante as entrevistas.

$\mathrm{Na}$ tabela 2, as plantas foram agrupadas conforme a indicação terapêutica, bem como foram indicados por letras, os órgãos das plantas citados nos questionários para o tratamento das doenças.

\section{RESULTADOS E DISCUSSÃO}

Neste levantamento foram obtidas informações sobre 148 espécies, pertencentes a 134 gêneros, distribuídas em 64 famílias. A família Compositae contribuiu com o maior número de espécies (17), seguida das famílias Labiatae (12), Myrtaceae (8) e Leguminosae (6). Entre as espécies mencionadas, ocorrem plantas nativas e introduzidas.

Através dos resultados obtidos nos questionários, observou-se que a utilização de plantas medicinais nos bairros de Santa Maria, onde foi efetuada a pesquisa, é um fato comprovado, como está demonstrado na FIGURA 2, onde a freqüência de uso contínuo (sempre) é de 50,7\%, ou seja, nos 404 questionários, 205 entrevistados usam continuamente plantas medicinais.

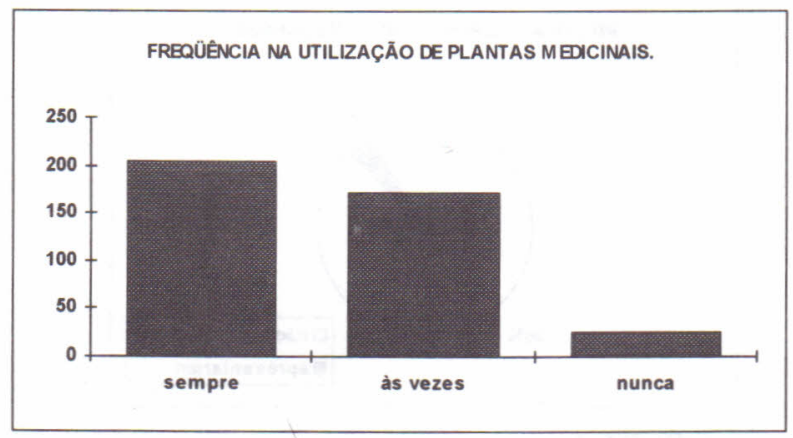

FIGURA 2 
A FIGURA 3 informa os locais de procedência das plantas, onde verifica-se que $76,0 \%$ das vias de obtenção são através de amigos e também pelo hábito do cultivo caseiro. Este fato indica que o uso de plantas é geralmente feito sem respaldo científico, intensificando, através desta automedicação, os erros na utilização das plantas medicinais.

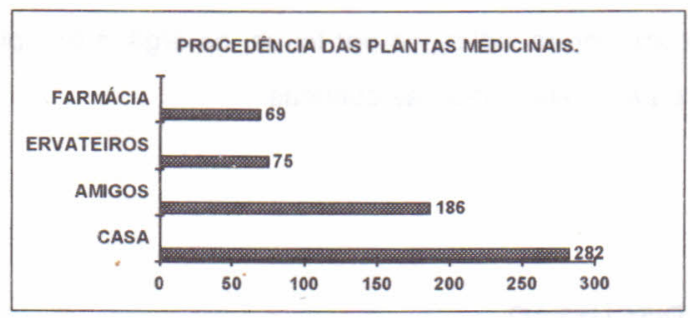

FIGURA 3

Na FIGURA 4 constata-se que a intoxicação por plantas medicinais não é um problema alarmante, sendo $4,0 \%$ os casos de intoxicação relatados. Porém, é importante salientar que as intoxicações, em sua maioria, foram constatadas devido ao uso excessivo de determinada planta, ou considerando os efeitos colaterais, como por exemplo, a carqueja (Baccharis articulata (Lam.) Pers.) usada para emagrecer, a qual provocou sintomas como súbita hipotensăo.

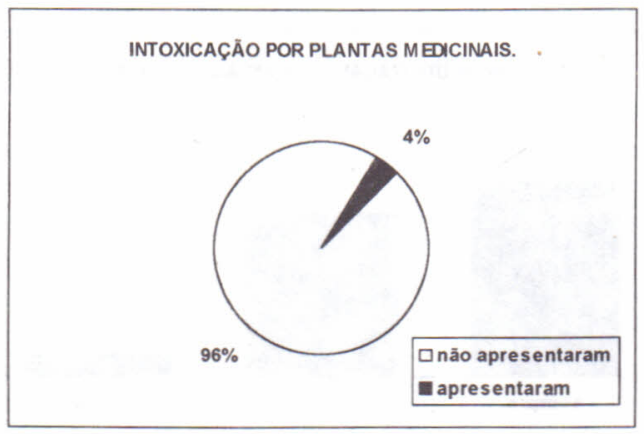

FIGURA 4 
As plantas medicinais devem ser tratadas pelo nome científico, para evitar trocas ou erros, pois existem muitas espécies diferentes conhecidas pelo mesmo nome popular, que pode variar de região para região. Com o fim de evitar problemas na identificação das plantas citadas nas entrevistas, a TABELA 1 apresenta o nome vulgar, o nome científico e a família de cada planta.

A TABELA 2 apresenta as indicações terapêuticas juntamente com o nome vulgar da planta e o(s) farmacógeno(s) utilizado(s). Nesta tabela, verifica-se que uma mesma planta tem indicações terapêuticas diferentes, como também o contrário pode ser observado.

Além dos aspectos descritos acima, constatou-se problemas como o uso de plantas misturadas com o chimarrão, que vem a ser um chá de erva-mate (llex paraguariensis St. Hil.), contendo diversas propriedades farmacológicas. Foi constatado também, a mistura de várias plantas usadas na preparação de chás e xaropes. SIMÕES et alii (1989) previnem que esta prática é perigosa, porque nem sempre o processo de preparação mais indicado é o mesmo para plantas diferentes e a combinação pode resultar em efeitos imprevisíveis.

As formas de utilização mais empregadas pela população são a infusão e a decocção. De acordo com MATOS (1989), infusão é quando, ao se preparar um chá, coloca-se água fervente em um recipiente contendo pedaços do vegetal, fecha-se o utensilio com uma tampa, e deixa-se descansar por cinco a dez minutos, sendo este método indicado para partes moles do vegetal como flores, folhas e frutos carnosos. Segundo o mesmo autor, decocção (ou cozimento) é a forma na qual as partes vegetais, geralmente duras como raízes, caules, sementes e frutos, são colocados em água fria e fervidos por cinco a dez minutos, com o recipiente tampado.

Constatou-se que a população está ciente destas informações, porém, não as põem em prática, utilizando-se do cozimento tanto para partes moles e duras do vegetal, sem considerar o tempo de fervura, sob a alegação de que na infusão, o preparado é inodoro e incolor e, portanto, ineficiente.

Além da infusão e decocção citadas acima, a população faz uso de alcoolaturas. Segundo Martins et alii (1995) esta preparação (realizada a frio), consiste em colocar a parte da planta medicinal dentro de um recipiente contendo 
álcool. Folhas, flores e partes tenras são picadas e ficam macerando por 10 a 12 horas, enquanto partes mais duras permanecem por 18 a 24 horas. $O$ recipiente deve permanecer em local fresco, protegido da luz direta e deverá ser agitado periodicamente. Os referidos autores não recomendam esta preparação para plantas com possibilidade de fermentações.

Outra preparação mencionada pelos entrevistados, é a de uso tópico, onde a planta é colocada diretamente nos locais afetados, como exemplo: o "sumo" (mucilagem) da folha de babosa (Aloe arborescens Mill.), o "leite" (látex) do quebra-pedra (Euphorbia serpens H.B.K.) e o tubérculo da batata-inglesa (Solanum tuberosum L.).

Observou-se ainda que algumas plantas são usadas por longos períodos, sem prescrição ou necessidade, como preventivas ou, simplesmente, como hábito.

TABELA 1: Nome popular, nome cientifico e família das plantas medicinais.

\begin{tabular}{|l|l|l|}
\hline \multicolumn{1}{|c|}{ NOME } & \multicolumn{1}{c|}{ NOME CIENTÍFICO } & \multicolumn{1}{c|}{ FAMÍLIA } \\
\hline abacateiro & Persea americana C. Bauh. & Lauraceae \\
\hline açoita-cavalo & Luehea divaricata Mart. & Tiliaceae \\
\hline agrião & Nasturtium officinale R.Br. & Cruciferae \\
\hline aipo & Apium graveolens L. & Umbelliferae \\
\hline alcachofra & Cynara scolymus L. & Compositae \\
\hline alcanfor & Artemisia camphorata Vill. & Compositae \\
\hline alecrim & Rosmarinus officinalis L. & Labiatae \\
\hline alfazema & Lavandula spica L. & Labiatae \\
\hline alho & Allium sativum L. & Liliaceae \\
\hline ameixeira & Eriobotrya japonica Lindl. & Rosaceae \\
\hline amoreira & Morus alba L. & Moraceae \\
\hline angico & Parapiptadenia rigida (Benth.) Bren. & Leguminosae \\
\hline anis & Ocimum selloi Benth. & Labiatae \\
\hline araçá & Psidium cattleianum Sab. & Myrtaceae \\
\hline araticum & Rollinia salicifolia Schlecht. & Annonaceae \\
\hline arnica & Chaptalia nutans (L.) Polak. & Compositae \\
\hline arruda & Ruta graveolens L. & Rutaceae \\
\hline avenca & Adianthum cuneatum L. & Pteridaceae \\
\hline babosa & Aloe arborescens Mill. & Liliaceae \\
\hline baicuru & Limonium brasiliense (Boiss.) Kuntze & Plumbaginaceae \\
\hline baldrame & Arctium lappa L. & Compositae \\
\hline bananeira & Musa sp. & Musaceae \\
\hline banana-do-mato & Bromelia anthiacantha Bert. & Bromeliaceae \\
\hline
\end{tabular}




\begin{tabular}{|c|c|c|}
\hline batata-doce & Ipomoea batatas (L.) Poir. & Convolvulaceae \\
\hline batata-inglesa & Solanum tuberosum L. & Solanaceae \\
\hline bergamoteira & Citrus reticulata Blanco & Rutaceae \\
\hline beringela & Solanum melongena $\mathrm{L}$. & Solanaceae \\
\hline boldo & Coleus barbatus (Andr.) Benth. & Labiatae \\
\hline $\begin{array}{l}\text { buchinha-do- } \\
\text { nordeste }\end{array}$ & Luffa operculata Cogn. & Cucurbitaceae \\
\hline cabelo-de-milho & Zea mays L. & Gramineae \\
\hline cabelo-de-porco & $\begin{array}{l}\text { Piptochaetium montevidensis (Spreng.) } \\
\text { Par. }\end{array}$ & Gramineae \\
\hline cabriúva & Myrocarpus frondosus Fr. All. & Leguminosae \\
\hline cacheta & $\begin{array}{l}\text { Didymopanax morototoni (Aubl.) } \\
\text { Decne. et Planch. }\end{array}$ & Araliaceae \\
\hline café & Coffea arabica L. & Rubiaceae \\
\hline caju & Anacardium occidentale L. & Anacardiaceae \\
\hline cambará & Gochnatia polymorpha (Less.) Cabr. & Compositae \\
\hline $\begin{array}{l}\text { camomila } \\
\text { (maçanilha) }\end{array}$ & Matricaria recutita $\mathrm{L}$. & Compositae \\
\hline cana-de-acúcar & Saccharum officinarum $\mathrm{L}$. & Gramineae \\
\hline canchalágua & Sizyrinchium sp. & Iridaceae \\
\hline cancorosa & Maytenus ilicifolia Mart. ex Reiss. & Celastraceae \\
\hline canela & Cinnamomum zeylanicum Breyn. & Lauraceae \\
\hline caninha-do-brejo & Hedychium coronarium Koen. & Zingiberaceae \\
\hline caqui & Diospyros kaki L. & Ebenaceae \\
\hline carambola & Averrhoa carambola L. & Oxalidaceae \\
\hline cardamomo & Alpinia speciosa (Wendl.) K. Schum. & Zingiberaceae \\
\hline carqueja & Baccharis articulata (Lam.) Pers. & Compositae \\
\hline $\begin{array}{l}\text { carrapicho- } \\
\text { rasteiro }\end{array}$ & Acanthospermum australe 0 . Kuntze & Compositae \\
\hline carvalhinho & Casearia sylvestris Sw. & Flacourtiaceae \\
\hline cáscara-sagrada & Rhamnus purshiana DC. & Rhamnaceae \\
\hline $\begin{array}{l}\text { catinga-de- } \\
\text { mulata }\end{array}$ & Tanacetum vulgare $\mathrm{L}$. & Compositae \\
\hline cebola & Allium cepa L. & Liliaceae \\
\hline cerejeira & Eugenia involucrata DC. & Myrtaceae \\
\hline chapéu-de-couro & Echinodorus grandiflorus Mich. & Alismataceae \\
\hline chuchu & Sechium edule Sw. & Cucurbitaceae \\
\hline cidreira & $\begin{array}{l}\text { Cymbopogon citratus (DC. ex Nees) } \\
\text { Stapf. }\end{array}$ & Gramineae \\
\hline cidró & Aloysia thriphylla (L'Herit.) Britt. & Verbenaceae \\
\hline cinamomo & Melia azedarach $\mathrm{L}$. & Meliaceae \\
\hline cipreste & Cupressus sp. & Cupressaceae \\
\hline côco-branco & Cocos nucifera L. & Palmae \\
\hline confrei & Symphytum officinale L. & Boraginaceae \\
\hline $\begin{array}{l}\text { coqueiro- } \\
\text { vermelho }\end{array}$ & $\begin{array}{l}\text { Syagrus romanzoffiana (Cham.) } \\
\text { Glassman }\end{array}$ & Palmae \\
\hline cordão-de-frade & Leonotis nepetaefolia R.Br. & Labiatae \\
\hline cortieeira & Erythrina crista-galli L. & Leguminosae \\
\hline couve & Brassica oleracea var. acephala L. & Cruciferae \\
\hline curunilho & Scutia buxifolia Reiss. & Rhamnaceae \\
\hline
\end{tabular}




\begin{tabular}{|c|c|c|}
\hline $\begin{array}{l}\text { douradinha-do- } \\
\text { campo }\end{array}$ & Waltheria douradinha St. Hil & Sterculiaceae \\
\hline erva-de-bicho & Polygonum punctatum Helliot & Polygonaceae \\
\hline $\begin{array}{l}\text { erva-de- } \\
\text { passarinho }\end{array}$ & Eubrachyon ambiguum Engl. & Loranthaceae \\
\hline erva-doce & Pimpinella anisum L. & Umbelliferae \\
\hline erva-guiné & Petiveria alliaceae $\mathrm{L}$. & Phytolacaceae \\
\hline erva-pombinha & Phyllanthus niruri L. & Euphorbiaceae \\
\hline erva-santa & Vernonia polyanthes Less. & Compositae \\
\hline erva-tostão & Boerhavia hirsuta Mart. & Nyctaginaceae \\
\hline eucalipto & Eucalyptus spp. & Myrtaceae \\
\hline fel-da-terra & Ionidium glutinosum Vent. & Violaceae \\
\hline figueira & Ficus carica L. & Moraceae \\
\hline flor-da-pedra & Usnea densirostra Tayl. & Usneaceae \\
\hline folha-da-fortuna & Kalanchoe pinnata Pers. & Crassulaceae \\
\hline funcho & Foeniculum vulgare Mill. & Umbelliferae \\
\hline garopá & Aloysia sellowii (Briq.) Mold. & Verbenaceae \\
\hline gengibre & Zingiber officinale Rosc. & Zingiberaceae \\
\hline gergelim & Sesamum indicum $\mathrm{L}$. & Pedaliaceae \\
\hline gervão & $\begin{array}{l}\text { Stachytarpheta cayennensis (Rich:) } \\
\text { Vahl. }\end{array}$ & Verbenaceae \\
\hline goiabeira & Psidium guajava $\mathrm{L}$. & Myrtaceae \\
\hline guaco & Mikania laevigata Sch. Bip. ex Baker & Compositae \\
\hline guajuvira & Patagonula americana $\mathrm{L}$. & Boraginaceae \\
\hline guanxuma & Sida rhombifolia L. & Malvaceae \\
\hline guavirova & Campomanesia xanthocarpa Berg. & Myrtaceae \\
\hline hortelã & Mentha sp. & Labiatae \\
\hline infalivina & Antemisia verlotorum Lam. & Compositae \\
\hline ipê-roxo & Tabebuia impetiginosa (Mart.) Standley & Bignoniaceae \\
\hline jambolão & Syzygium cuminii Skeels & Myrtaceae \\
\hline $\begin{array}{l}\text { japecanga } \\
\text { (salsaparrilha) }\end{array}$ & Smilax sp. & Liliaceae \\
\hline jurubeba & Solanum guaraniticum L. & Solanaceae \\
\hline $\begin{array}{l}\text { lágrima-de- } \\
\text { Nossa-Senhora }\end{array}$ & Coix lacrima-jobi $\mathrm{L}$. & Gramineae \\
\hline laranjeira & Citrus sinensis (L.) Osb. & Rutaceae \\
\hline levante & Mentha citrata Ehrart & Labiatae \\
\hline limoeiro & Citrus limon (L.) Burn. & Rutaceae \\
\hline losna & Artemisia absinthium L. & Compositae \\
\hline lucera (quitoco) & Pluchea sagittalis (Lam.) Cabrera & Compositae \\
\hline macieira & Malus sylvestris Mill. & Rosaceae \\
\hline malva & Malva sylvestris L. & Malvaceae \\
\hline mamoeiro & Carica papaya L. & Caricaceae \\
\hline $\begin{array}{l}\text { mamica-de- } \\
\text { cadela }\end{array}$ & Zanthoxylum rhoifolium Lam. & Rutaceae \\
\hline mangueira & Mangifera indica $L$. & Anacardiaceae \\
\hline mangerona & Majorana hortensis Moench. & Labiatae \\
\hline manjericăo & Ocimum basilicum L. & Labiatae \\
\hline manto-de-viúva & Setcreasea purpurea Boom. & Commelinaceae \\
\hline maracujá & Passiflora alata Dryander & Passifloraceae \\
\hline
\end{tabular}




\begin{tabular}{|c|c|c|}
\hline maravilha & Mirabilis jalapa L. & Nyctaginaceae \\
\hline marcela & Achyrocline satureioides (Lam.) DC. & Compositae \\
\hline marmelo & Cydonia oblonga Mill. & Rosaceae \\
\hline mastruço & Lepidium sativum L. & Cruciferae \\
\hline melissa & Melissa officinalis L. & Labiatae \\
\hline $\begin{array}{l}\text { mil-folhas } \\
\text { (pronto-alívio) }\end{array}$ & Achillea millefolium L. & Compositae \\
\hline murta & $\begin{array}{l}\text { Blepharocalyx salicifolius var. tweediei } \\
\text { (Hook. et Arn.) Legr. }\end{array}$ & Myrtaceae \\
\hline nabo & $\begin{array}{l}\text { Brassica napus var. napobrassica (L.) } \\
\text { Petern. }\end{array}$ & Cruciferae \\
\hline nogueira & Carya illinoensis (Wangh.) Koch. & Juglandaceae \\
\hline noz-moscada & Myristica fragrans Houtt. & Myristicaceae \\
\hline oliveira & Olea europaea var. sativa DC. & Oleaceae \\
\hline pata-de-vaca & Bauhinia forficata L. K. & Leguminosae \\
\hline pariparoba & Piper gaudichaudianum Kunth & Piperaceae \\
\hline parreira & Vitis vinifera $\mathrm{L}$. & Vitaceae \\
\hline pau-ferro & Caesalpinia ferrea Mart. & Leguminosae \\
\hline pessegueiro & Prunus persica (L.) Batsch. & Rosaceae \\
\hline picão & Bidens pilosa $\mathrm{L}$. & Compositae \\
\hline pitangueira & Eugenia uniflora L. & Myrtaceae \\
\hline poaia (poalho) & Borreria verticilatta (L.) Meyer & Rubiaceae \\
\hline poejo & Cunila microcephala Benth. & Labiatae \\
\hline quebra-pedra & Euphorbia serpens H.B.K. & Euphorbiaceae \\
\hline quiabo & Hibiscus esculentus L. & Malvaceae \\
\hline romã & Punica granatum L. & Punicaceae \\
\hline rosa-branca & Rosa sp. & Rosaceae \\
\hline sabugueiro & Sambucus australis Cham. et Schlecht. & Caprifoliaceae \\
\hline salsa & Petroselinum crispum (Mill.) Nym. & Umbelliferae \\
\hline sálvia & Salvia officinalis L. & Labiatae \\
\hline samambaia-roxa & Adiantopsis chlorophylla (Sw.) Fee & Polypodiaceae \\
\hline saúde-da-mulher & Abutilon molle (Ort.) Sweet. & Malvaceae \\
\hline sene & Senna corymbosa (Lam.) Irw. et Barn. & Leguminosae \\
\hline sete-sangrias & Cuphea ingrata Cham. & Lythraceae \\
\hline taiuiá & Cayaponia martiana (Cogn.) Cogn. & Cucurbitaceae \\
\hline tarumã & Vitex megapotamica (Spr.) Mold. & Verbenaceae \\
\hline tomateiro & Lycopersicom esculentum Mill. & Solanaceae. \\
\hline transagem & Plantago australis Lam. & Plantaginaceae \\
\hline tuna & Opuntia sp. & Cactaceae \\
\hline urtiga & Urera baccifera Gaud. & Urticaceae \\
\hline uva-do-mato & Odontocarya acuparata Miers & Menispermaceae \\
\hline violeta & Viola odorata L. & Violaceae \\
\hline
\end{tabular}


TABELA 2. Indicação terapêutica e farmacógeno das plantas medicinais.

\begin{tabular}{|c|c|c|}
\hline $\begin{array}{l}\text { INDICAÇÃO } \\
\text { TERAPÊUTICA }\end{array}$ & PLANTA & FARMACÓGENO \\
\hline 1. Afta & $\begin{array}{l}\text { arnica, batata-doce, } \\
\text { transagem. }\end{array}$ & a. Folha \\
\hline 2. Analgésico & $\begin{array}{l}\text { aipo (e), anis (a,d), batata- } \\
\text { inglesa (c), bergamoteira } \\
\text { (a,d), boldo (a), camomila } \\
\text { (b), caqui (a), cardamomo } \\
(\mathrm{a}, \mathrm{b}) \text {, carqueja }(\mathrm{c}) \text {, cidreira } \\
\text { (a), funcho (a,d), gervão } \\
\text { (a), infalivina (a), laranjeira } \\
\text { (a,d), levante (a), losna (a), } \\
\text { malva (e), manjericão (a), } \\
\text { maravilha (a), marcela (b), } \\
\text { melissa (a), mil-folhas (a), } \\
\text { sálvia (a). }\end{array}$ & $\begin{array}{l}\text { a. Folha. } \\
\text { b. Flor. } \\
\text { c. Caule. } \\
\text { d. Fruto. } \\
\text { e. Toda planta. }\end{array}$ \\
\hline 3. Anemia & $\begin{array}{l}\text { açoita-cavalo (a), nogueira } \\
\text { (b), pau-ferro (a). }\end{array}$ & $\begin{array}{l}\text { a. Caule (casca). } \\
\text { b. Toda planta. }\end{array}$ \\
\hline 4. Anorexia & carqueja. & a. Caule \\
\hline 5. Antidiarrréico & $\begin{array}{l}\text { carqueja (b), erva-tostão } \\
(a, c), \text { fel-da-terra }(c) \text {, } \\
\text { goiabeira }(a, f) \text {, marmelo } \\
(a, e), \text { noz-moscada }(g) \text {, } \\
\text { parreira }(a), \text { pitangueira } \\
(a, b, f), \text { poaia }(a), \text { romã }(e) .\end{array}$ & $\begin{array}{l}\text { a. Folha. } \\
\text { b. Caule. } \\
\text { c. Raiz. } \\
\text { d. Caule (casca). } \\
\text { e. Fruto (casca). } \\
\text { f. Broto (gemas). } \\
\text { g. Semente. }\end{array}$ \\
\hline 6. Antiespasmódico & $\begin{array}{l}\text { alfazema (a), camomila (b), } \\
\text { canela (d), erva-doce (c), } \\
\text { funcho (a,c), hortelä (a), } \\
\text { mangerona (a), melissa } \\
\text { (a), mil-folhas (a), saúde- } \\
\text { da-mulher (a). }\end{array}$ & $\begin{array}{l}\text { a. Folha. } \\
\text { b. Flor. } \\
\text { c. Fruto. } \\
\text { d. Caule (casca). }\end{array}$ \\
\hline 7. Anti-hemorrágico & mil-folhas. & a. Folha \\
\hline 8. Antiinflamatório & 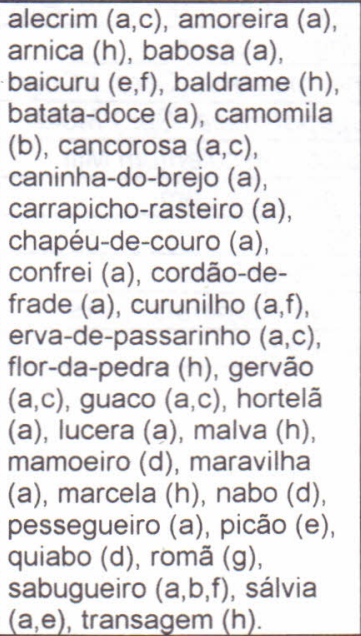 & $\begin{array}{l}\text { a. Folha. } \\
\text { b. Flor. } \\
\text { c. Caule. } \\
\text { d. Fruto. } \\
\text { e. Raiz. } \\
\text { f. Caule (casca). } \\
\text { g. Fruto (casca). } \\
\text { h. Toda planta. }\end{array}$ \\
\hline
\end{tabular}




\begin{tabular}{|c|c|c|}
\hline 9. Anti-reumático & $\begin{array}{l}\text { abacateiro (c), alcanfor } \\
\text { (a,d), babosa (a), } \\
\text { carvalhinho (a), chapéu- } \\
\text { de-couro (a), confrei (a), } \\
\text { manjericão (a), marcela } \\
\text { (d), mastruço (d), pau-ferro } \\
\text { (b), samambaia-roxa (d), } \\
\text { tuna (a). }\end{array}$ & $\begin{array}{l}\text { a. Folha. } \\
\text { b. Caule (casca). } \\
\text { c. Fruto. } \\
\text { d. Toda planta. }\end{array}$ \\
\hline 10. Antitumoral & $\begin{array}{l}\text { babosa }(a), \text { ipê-roxo }(c) \\
\text { macieira }(a, b, c)\end{array}$ & $\begin{array}{l}\text { a. Folha. } \\
\text { b. Fruto. } \\
\text { c. Caule (casca). }\end{array}$ \\
\hline 11. Antitussígeno & $\begin{array}{l}\text { açoita-cavalo (e), agrião } \\
(f) \text {, alecrim }(a, c) \text {, amexeira } \\
\text { (a), anis }(a, c) \text {, angico }(e), \\
\text { avenca }(a) \text {, cambará }(a, e) \text {, } \\
\text { figueira }(a, d) \text {, funcho }(a, d) \text {, } \\
\text { guaco }(a) \text {, laranjeira }(a, b, d \text {, } \\
\text { e), marcela (f), mastruço } \\
\text { (f), poejo }(a) \text {, sálvia }(a) \text {, } \\
\text { transagem (f). }\end{array}$ & $\begin{array}{l}\text { a. Folha. } \\
\text { b. Flor. } \\
\text { c. Caule. } \\
\text { d. Fruto. } \\
\text { e. Caule (casca). } \\
\text { f. Toda planta. }\end{array}$ \\
\hline 12. Calmante & $\begin{array}{l}\text { alecrim }(a), \text { anis }(a, c) \text {, } \\
\text { batata-doce }(a), \\
\text { bergamoteira }(a, d) \text {, } \\
\text { camomila }(b) \text {, carqueja }(c) \text {, } \\
\text { chuchu }(a) \text {, cidreira }(g) \text {, } \\
\text { cidró }(a), \text { côco-branco }(f), \\
\text { confrei }(a), \text { erva-doce }(d) \text {, } \\
\text { hortelã }(a) \text {, laranjeira }(a) \text {, } \\
\text { macieira }(a, d), \text { mangerona } \\
\text { (a), manjericão (a), } \\
\text { maracujá }(a, d), \text { marcela } \\
\text { (g), melissa }(a) \text {, } \\
\text { pitangueira }(a) \text {. }\end{array}$ & $\begin{array}{l}\text { a. Folha. } \\
\text { b. Flor. } \\
\text { c. Caule. } \\
\text { d. Fruto. } \\
\text { e. Broto (gemas). } \\
\text { f. Fruto (casca). } \\
\text { g. Toda planta. }\end{array}$ \\
\hline 13. Carminativo & $\begin{array}{l}\text { erva-de-bicho (a), erva- } \\
\text { doce }(b) \text {, figueira }(a, b) \\
\text { funcho }(a, b) \text {, hortelã }(a) \text {. }\end{array}$ & $\begin{array}{l}\text { a. Folha. } \\
\text { b. Fruto. }\end{array}$ \\
\hline 14. Cicatrizante & $\begin{array}{l}\text { arnica (d), babosa (a), } \\
\text { carvalhinho (a), cinamomo } \\
\text { (c), confrei (a), erva-de- } \\
\text { bicho (a), erva-santa (a), } \\
\text { folha-da-fortuna (a), malva } \\
\text { (d), mastruço (b), } \\
\text { sabugueiro }(a, b, c) \text {. }\end{array}$ & $\begin{array}{l}\text { a. Folha. } \\
\text { b. Flor. } \\
\text { c. Caule (casca). } \\
\text { d. Toda planta. }\end{array}$ \\
\hline 15. Colagogo & $\begin{array}{l}\text { abacateiro (a), alcachofra } \\
\text { (a), arnica (f), boldo (a,c), } \\
\text { cacheta (a), camomila (b), } \\
\text { carqueja (c), catinga-de- } \\
\text { mulata }(a, c) \text {, erva-tostão } \\
(a, e) \text {, garopá (a), } \\
\text { guanxuma (f), infalivina } \\
(a, c) \text {, jurubeba (a,e), losna } \\
\text { (a,c), malva (f), mangerona } \\
\text { (a,c), marcela (f), nogueira } \\
\text { (a), noz-moscada (d), } \\
\text { pariparoba (a), uva-do- } \\
\text { mato (f). }\end{array}$ & $\begin{array}{l}\text { a. Folha. } \\
\text { b. Flor. } \\
\text { c. Caule. } \\
\text { d. Fruto. } \\
\text { e. Raiz. } \\
\text { f. Toda planta. }\end{array}$ \\
\hline
\end{tabular}




\begin{tabular}{|c|c|c|}
\hline 16. Conjuntivite & $\begin{array}{l}\text { erva-santa (a), rosa-branca } \\
\text { (b). }\end{array}$ & $\begin{array}{l}\text { a. Folha. } \\
\text { b. Flor (pétalas). }\end{array}$ \\
\hline 17. Contusão & $\begin{array}{l}\text { alcanfor (c), alecrim }(a, b) \text {, } \\
\text { catinga-de-mulata }(a, b) \text {, } \\
\text { erva-de-passarinho }(c), \\
\text { eucalipto }(a) \text {, mastruço }(c)\end{array}$ & $\begin{array}{l}\text { a. Folha. } \\
\text { b. Caule. } \\
\text { c. Toda planta. }\end{array}$ \\
\hline 18. "Cravo" & quebra-pedra. & a. Látex (leite). \\
\hline 19. Depurativo & $\begin{array}{l}\text { açoita-cavalo (e), boldo } \\
\text { (a,b), douradinha-do- } \\
\text { campo (f), gerväo (f), } \\
\text { japecanga (a,b,d), } \\
\text { jurubeba }(a) \text {, limoeiro } \\
(a, c, e) \text {, transagem (f). }\end{array}$ & $\begin{array}{l}\text { a. Folha. } \\
\text { b. Caule. } \\
\text { c. Fruto. } \\
\text { d. Raiz. } \\
\text { e. Caule (casca). } \\
\text { f. Toda planta. }\end{array}$ \\
\hline $\begin{array}{l}\text { 20. Descamação da } \\
\text { pele }\end{array}$ & $\begin{array}{l}\text { arnica }(a), \text { cipreste }(b), \\
\text { malva (a). }\end{array}$ & $\begin{array}{l}\text { a. Folha. } \\
\text { b. Toda planta. }\end{array}$ \\
\hline 21.Digestivo & 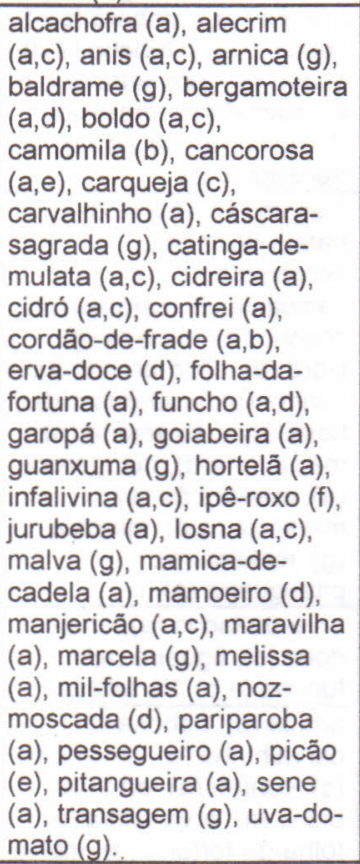 & $\begin{array}{l}\text { a. Folha. } \\
\text { b. Flor. } \\
\text { c. Caule. } \\
\text { d. Fruto. } \\
\text { e. Raiz. } \\
\text { f. Caule (casca). } \\
\text { g. Toda planta. }\end{array}$ \\
\hline 22. Diurético & $\begin{array}{l}\text { abacateiro (d), amoreira } \\
\text { (a), arnica (f), batata-doce } \\
\text { (a), cabelo-de-milho (e), } \\
\text { caninha-do-brejo (a), } \\
\text { carvalhinho (a), cidreira } \\
\text { (a), erva-pombinha (f), } \\
\text { folha-da-fortuna (a), } \\
\text { guanxuma (f), guavirova } \\
\text { (a), mangueira (a), pata- } \\
\text { de-vaca (a), picão (c), } \\
\text { salsa (a,c), tomateiro (a), } \\
\text { transagem (f). }\end{array}$ & $\begin{array}{l}\text { a. Folha. } \\
\text { b. Caule. } \\
\text { c. Raiz. } \\
\text { d. Fruto (caroço). } \\
\text { e. Estigma de milho } \\
\text { ("cabelo-de-milho"). } \\
\text { f. Toda planta. }\end{array}$ \\
\hline 23. Emagrecimento & $\begin{array}{l}\text { alcachofra (a), carqueja } \\
\text { (c), guavirova (a), } \\
\text { jambolão (a), jurubeba (a), } \\
\text { mangueira (a), nabo (b). }\end{array}$ & $\begin{array}{l}\text { a. Folha. } \\
\text { b. Fruto. } \\
\text { c. Toda planta. }\end{array}$ \\
\hline
\end{tabular}




\begin{tabular}{|c|c|c|}
\hline 24. Emenagogo & arruda (a), canela (b). & $\begin{array}{l}\text { a. Folha. } \\
\text { b. Caule (casca). }\end{array}$ \\
\hline 25. Emético & couve. & a. Folha. \\
\hline 26. Estimulante & gengibre (b), limoeiro (a). & $\begin{array}{l}\text { a. Folha. } \\
\text { b. Caule (rizoma). }\end{array}$ \\
\hline 27. Expectorante & $\begin{array}{l}\text { erva-de-passarinho }(a, b), \\
\text { eucalipto (a), guaco (a), } \\
\text { poejo (a). }\end{array}$ & $\begin{array}{l}\text { a. Folha. } \\
\text { b. Caule. }\end{array}$ \\
\hline 28. Extração de veneno & cebola. & a. Caule (bulbo). \\
\hline 29.Febrífugo & $\begin{array}{l}\text { aipo (a), cidreira (a), erva- } \\
\text { doce }(b), \text { marcela }(e) \text {, } \\
\text { sabugueiro }(a, d) \text {, sálvia } \\
(a, c) \text {. }\end{array}$ & $\begin{array}{l}\text { a. Folha. } \\
\text { b. Fruto. } \\
\text { c. Raiz. } \\
\text { d. Caule (casca). } \\
\text { e. Toda planta. }\end{array}$ \\
\hline 30. Gota & carqueja (b), guavirova (a). & $\begin{array}{l}\text { a. Folha. } \\
\text { b. Caule. }\end{array}$ \\
\hline 31.Gripe & 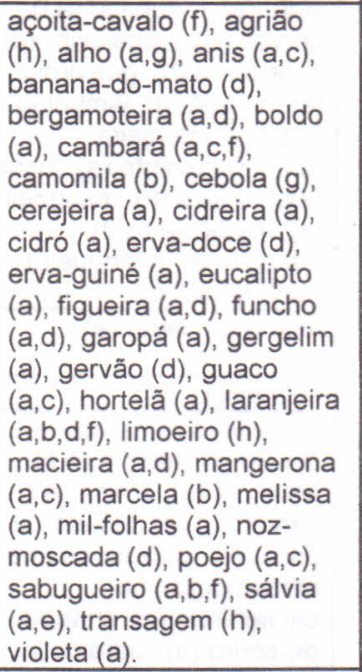 & $\begin{array}{l}\text { a. Folha. } \\
\text { b. Flor. } \\
\text { c. Caule. } \\
\text { d. Fruto. } \\
\text { e. Raiz. } \\
\text { f. Caule (casca). } \\
\text { g. Caule (bulbo). } \\
\text { h. Toda planta. }\end{array}$ \\
\hline 32. Hemorróida & $\begin{array}{l}\text { erva-de-bicho (a), erva-de- } \\
\text { passarinho }(a, b) \text {, } \\
\text { mangueira (a), maravilha } \\
\text { (a). }\end{array}$ & $\begin{array}{l}\text { a. Folha. } \\
\text { b. Caule. }\end{array}$ \\
\hline 33. Hepatite & salsa. & a. Raiz. \\
\hline 34. Hipocolesteremiante & $\begin{array}{l}\text { alcachofra (a), amoreira } \\
\text { (a), anis }(a, b) \text {, carqueja (b), } \\
\text { carvalhinho (a), folha-da- } \\
\text { fortuna }(a) \text {, gervão }(a, b) \text {, } \\
\text { guanxuma (h), guavirova } \\
\text { (a), jamboläo }(a, c, e, g) \text {, } \\
\text { japecanga (a,b,d), macieira } \\
(a, c, f) \text {, mangueira (a), } \\
\text { oliveira (a), pau-ferro (e), } \\
\text { picão (d), sete-sangrias } \\
\text { (e). }\end{array}$ & $\begin{array}{l}\text { a. Folha. } \\
\text { b. Caule. } \\
\text { c. Fruto. } \\
\text { d. Raiz. } \\
\text { e. Caule (casca). } \\
\text { f. Fruto (casca). } \\
\text { g. Fruto (caroço). } \\
\text { h. Toda planta. }\end{array}$ \\
\hline
\end{tabular}




\begin{tabular}{|c|c|c|}
\hline 35. Hipoglicemiante & $\begin{array}{l}\text { batata-doce (a), beringela } \\
\text { (c), café (c), caju (a), } \\
\text { carambola }(a) \text {, carvalhinho } \\
\text { (a), curunilho }(a, e), \\
\text { guanxuma }(g), \text { guavirova } \\
\text { (a), jamboläo }(a, c, e, f), \\
\text { japecanga }(a, b, d), \\
\text { mangueira }(a), \text { marcela }(g), \\
\text { pata-de-vaca (a), pau-ferro } \\
\text { (e), taiuiá (e). }\end{array}$ & $\begin{array}{l}\text { a. Folha. } \\
\text { b. Caule. } \\
\text { c. Fruto. } \\
\text { d. Raiz. } \\
\text { e. Caule (casca). } \\
\text { f. Fruto (caroço). } \\
\text { g. Toda planta. }\end{array}$ \\
\hline 36. Hipotensor & 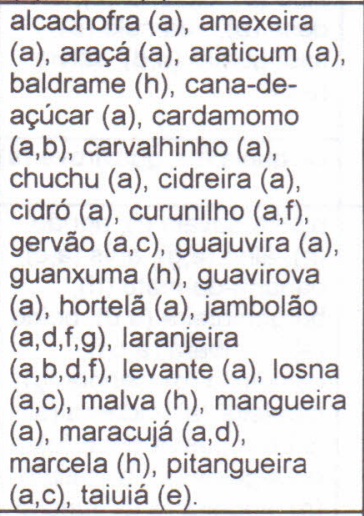 & $\begin{array}{l}\text { a. Folha. } \\
\text { b. Flor. } \\
\text { c. Caule. } \\
\text { d. Fruto. } \\
\text { e. Raiz. } \\
\text { f. Caule (casca). } \\
\text { g. Fruto (caroço). } \\
\text { h. Toda planta. }\end{array}$ \\
\hline 37. Laxante & aipo $(a, b)$, sene $(a)$ & $\begin{array}{l}\text { a. Folha. } \\
\text { b. Raiz. }\end{array}$ \\
\hline 38. Olheira & camomila. & a. Flor. \\
\hline $\begin{array}{l}\text { 39. Problemas } \\
\text { circulatórios }\end{array}$ & $\begin{array}{l}\text { abacateiro }(a, g) \text {, açoita- } \\
\text { cavalo }(f) \text {, alecrim }(a, c), \\
\text { araticum }(a) \text {, arnica }(a, c, e) \text {, } \\
\text { bananeira }(d) \text {, cabelo-de- } \\
\text { porco }(h) \text {, cancorosa }(a, e) \text {, } \\
\text { cardamomo }(a, b) \text {, } \\
\text { carvalhinho }(a) \text {, chapéu- } \\
\text { de-couro }(a) \text {, cidreira }(a) \text {, } \\
\text { coqueiro-vermelho (f), } \\
\text { curunilho }(a, f) \text {, douradinha- } \\
\text { do-campo }(h) \text {, erva-de- } \\
\text { passarinho }(a, c) \text {, funcho } \\
\text { (a,d), gervão }(a, c) \text {, } \\
\text { guanxuma }(h), \text { guavirova } \\
\text { (a), ipê-roxo (f), japecanga } \\
\text { (a,c,e), nogueira (a), pau- } \\
\text { ferro (f), taiuiá }(e) \text {, tarumã } \\
\text { (f). }\end{array}$ & $\begin{array}{l}\text { a. Folha. } \\
\text { b. Flor. } \\
\text { c. Caule. } \\
\text { d. Fruto.' } \\
\text { e. Raiz. } \\
\text { f. Caule (casca). } \\
\text { g. Fruto (caroço). } \\
\text { h. Toda planta. }\end{array}$ \\
\hline
\end{tabular}




\begin{tabular}{|c|c|c|}
\hline 40. Problemas renais & $\begin{array}{l}\text { abacateiro (a,c), arnica } \\
\text { (a,b,d), cabelo-de-porco } \\
\text { (f), canchalágua (f), } \\
\text { carvalhinho (a), } \\
\text { douradinha-do-campo (f), } \\
\text { erva-pombinha (f), erva- } \\
\text { tostão (a,d), flor-da-pedra } \\
\text { (f), guanxuma (f), } \\
\text { japecanga (a,d), lágrima- } \\
\text { de-nossa-senhora (a), } \\
\text { manto-de-viúva (f), } \\
\text { pessegueiro (a), quebra- } \\
\text { pedra (f), salsa (d), } \\
\text { transagem (f), urtiga (d), } \\
\text { uva-do-mato (f). }\end{array}$ & $\begin{array}{l}\text { a. Folha. } \\
\text { b. Caule. } \\
\text { c. Fruto. } \\
\text { d. Raiz. } \\
\text { e. Fruto (caroço). } \\
\text { f. Toda planta. }\end{array}$ \\
\hline 41. Sarampo & sabugueiro. & \begin{tabular}{|l|} 
a. Folha. \\
b. Flor. \\
c. Caule. \\
\end{tabular} \\
\hline 42. Sedativo. & corticeira. & a. Caule (casca). \\
\hline 43. Sinusite & $\begin{array}{l}\text { baicurú }(b, c) \text {, buchinha-do- } \\
\text { nordeste }(a) \text {, cabriúva }(c) \text {. }\end{array}$ & $\begin{array}{l}\text { a. Fruto. } \\
\text { b. Raiz. } \\
\text { c. Caule (casca). }\end{array}$ \\
\hline 44. Taquicardia & $\begin{array}{l}\text { cardamomo (a,b), jambolão } \\
(\mathrm{a}) \text {, laranjeira }(a), \\
\text { mangerona }(a, c) .\end{array}$ & $\begin{array}{l}\text { a. Folha. } \\
\text { b. Flor. } \\
\text { c. Caule. }\end{array}$ \\
\hline 45. Tétano & quiabo. & a.Fruto. \\
\hline 46. Úlcera & $\begin{array}{l}\text { cancorosa (a,c), jurubeba } \\
\text { (a), mamica-de-cadela (a), } \\
\text { mamoeiro (b). }\end{array}$ & $\begin{array}{l}\text { a. Folha. } \\
\text { b. Fruto. } \\
\text { c. Raiz. }\end{array}$ \\
\hline 47. Vermífugo & hortelã, losna. & a. Folha. \\
\hline 48. Verrugas & quebra-pedra. & a. Látex (leite). \\
\hline
\end{tabular}

\section{CONCLUSÃO}

Com este trabalho concluiu-se que o uso de plantas medicinais é muito difundido, no entanto, apesar de existirem muitas práticas coerentes, outras são motivos de preocupação como:

1. O uso excessivo das plantas medicinais.

2. Preparação incorreta das plantas.

3. Falta de conhecimento dos efeitos colaterais que poderão ser provocados pelo uso das plantas medicinais.

4. A mistura indiscriminada de plantas.

5. O uso de plantas misturadas com o chimarrão. 
Os resultados obtidos demonstram que as informações populares são valiosas, porém, ressalta-se a importância de estudos científicos que venham comprovar ou não estas informações, a fim de poder servir à população com maior segurança e eficácia.

\section{BIBLIOGRAFIA}

1. MARTINS, E.R. et alii. Plantas medicinais. Viçosa: Imprensa Universitária, 1995. 220 p.

2. MATOS, F.J.A. Plantas medicinais: guia de seleção e emprego de plantas medicinais do Nordeste do Brasil. Fortaleza: IOCE, v.1, 1989. 164 p.

3. SIMÕES, C.M.O. et alii. Plantas da medicina popular do Rio Grande do Sul. 3. ed. Porto Alegre: Editora da Universidade/UFRGS, 1989. 174 p. 